\title{
11-O-acetylcyathatriol inhibits MAPK/p38-mediated inflammation in LPS-activated RAW 264.7 macrophages and has a protective effect on ethanol-induced gastric injury
}

\author{
XIN JIN $^{1 *}$, JUNJIE HAN $^{2 *}$, SHUXIAN YANG ${ }^{1}$, YUAN HU $^{1}$, HONGWEI LIU ${ }^{2}$ and FENG ZHAO ${ }^{1}$ \\ ${ }^{1}$ Key Laboratory of Molecular Pharmacology and Drug Evaluation, Ministry of Education of China, \\ School of Pharmacy, Yantai University, Yantai, Shandong 264005; ${ }^{2}$ State Key Laboratory of Mycology, \\ Institute of Microbiology, Chinese Academy of Sciences, Beijing 100080, P.R. China
}

Received May 30, 2015; Accepted April 4, 2016

DOI: $10.3892 / \mathrm{mmr} .2016 .5305$

\begin{abstract}
The present study investigated the effects of 11- $O$-acetylcyathatriol, a natural cyathane diterpene, on the release of inflammatory mediators and on the activation of the nuclear factor $(\mathrm{NF})-\kappa \mathrm{B}$ or the mitogen-activated protein kinase (MAPK) transduction pathways in lipopolysaccharide (LPS)-activated macrophages. MTT was used to evaluate the cytotoxicity. A Griess assay was used to determine the production of nitrous oxide (NO). The levels of tumor necrosis factor (TNF)- $\alpha$ and interleukin (IL)-6 were determined using ELISA kits. The protein expression levels of inducible nitric oxide synthase (iNOS), cyclooxygenase (COX)-2, phosphorylated (p)-extracellular signal-regulated kinase (ERK1/2), p-J-N-terminal kinase (JNK), p-p38 and inhibitor of $\mathrm{NF} \kappa \mathrm{B}(\mathrm{I} \kappa \mathrm{B})-\alpha$ were detected using western blot analysis. 11- $O$-acetylcyathatriol significantly inhibited the overproduction of NO and the release of IL-6, but had no inhibitory effect on the release of TNF- $\alpha$. It also significantly downregulated the high expression levels of iNOS and COX-2 induced by LPS. In addition, it markedly inhibited the phosphorylation of the MAPK/p38 protein, but only exhibited weak inhibition on
\end{abstract}

Correspondence to: Professor Feng Zhao, Key Laboratory of Molecular Pharmacology and Drug Evaluation, Ministry of Education of China, School of Pharmacy, Yantai University, 30 Qingquan Road of Laishan, Yantai, Shandong 264005, P.R. China E-mail: ytuzhaofeng@163.com

Professor Hongwei Liu, State Key Laboratory of Mycology, Institute of Microbiology, Chinese Academy of Sciences, 1 Beichen West Road, Chaoyang, Beijing 100080, P.R. China

E-mail: liuhw@im.ac.cn

${ }^{*}$ Contributed equally

Key words: nidulariaceae, Cyathus africanus, cyathane diterpenoid, 11- $O$-acetylcyathatriol, anti-inflammatory effect the phosphorylation of the ERK1/2 and JNK proteins, and the degradation of the I $\kappa \mathrm{B}-\alpha$ protein. The possible protective effect of 11- $O$-acetylcyathatriol on ethanol-induced gastric injury was also examined using an in vivo animal experiment. Following gavage administration, it showed an important protective effect on ethanol-induced gastric mucosal injury in rats. These results suggested the possibility that the anti-inflammatory effect of 11- $O$-acetylcyathatriol was predominantly due to the inhibition of iNOS and COX-2 proteins, and may be associated with the MAPK/p38 transduction pathway, but not the $\mathrm{NF}-\kappa \mathrm{B}$ transduction pathway. These findings provide an explanation for the underlying mechanism of anti-inflammatory action of 11-O-acetylcyathatriol, which may assist with its clinical application and future development.

\section{Introduction}

Cyathus is a genus of fungi in the Nidulariaceae family, which is collectively known as bird's nest fungi (1). The Cyathus genus produces high levels of cyathane-type diterpenoids with a unique tricyclic skeleton (2-4). Cyathane-type diterpenoids represent a group of natural products with substantial diversity in chemical structure and bioactivities (5-7). The identification of cyathin A3 and allocyathin B3 from the liquid culture of Cyathus helenae in 1972 represents the first identification of cyathane-type diterpenoids (2). There have been continued investigations into these particular natural compounds, leading to the purification and structural elucidation of a number of cyathane-type diterpenoids from the Cyathus genus, including $C$. helenae (2,8-10), C. africanus (11), C. striatius (12) and C. earlei (13). However, until now, reports on the bioactivities of these cyathane diterpenoids remain limited, with the exception of a small number of studies on the neurotrophic activities of striatoids A-F isolated from cultures of the fungus Cyathus striatus (14), and identification of cyathuscavins A, B and C as free-radical scavengers with DNA protective activity (15).

In our previous study, eight cyathane-type diterpenoids (cyathatriol, 11-O-acetylcyathatriol, cyathins D-H and 
neosarcodonin O) were identified from the solid culture of C. africanus (16). The bioassay results firstly indicated that 11- $O$-acetylcyathatriol exhibits potent inhibitory effects against nitric oxide (NO) secretion in lipopolysaccharide (LPS)-activated macrophages. 11- $O$-acetylcyathatriol is a cyathane diterpenoid with the highest yield from C.africanus, and the previous bioassay results provide direct evidence to support its possible anti-inflammatory property. In order to confirm its anti-inflammatory effect and to clarify the molecular mechanism, the in vitro and in vivo anti-inflammatory activities, and the regulatory effect on the inflammatory signal transduction pathway of 11-O-acetylcyathatriol were further investigated and reported in the present study.

\section{Materials and methods}

Fungal material. The fungal strain was isolated from the fruiting body of $C$. africanus, and identified by Professor Yuhui Chen (Institute of Microbiology, Chinese Academy of Sciences, Beijing, China). The fungus was deposited in the China General Microbiological Culture Collection (CGMCC 5.1117).

Strain fermentation and isolation of 11-O-acetylcyathatriol. Strain fermentation of $C$. africanus was performed, as described previously (16). The fermented substrate was extracted using ethyl acetate (Tianjin Damao Chemical Reagent Factory, Tianjin, China) by exhaustive maceration (5 1, three times), and the solvent was then evaporated under reduced pressure to obtain the crude extract $(66.5 \mathrm{~g})$. The residue was resuspensed in $400 \mathrm{ml}$ of water and partitioned with an equal volume of $\mathrm{CHCl}_{3}$ (Tianjin Damao Chemical Reagent Factory) to yield the $\mathrm{CHCl}_{3}$-soluble fraction (46 g). The $\mathrm{CHCl}_{3}$-soluble fraction $(30 \mathrm{~g}$ ) was subjected to silica gel column chromatography and eluted with a gradient of $n$-hexane-ethyl acetate (v/v, 100:0-100:35) and dichloromethane-acetone (v/v, 100:2-0:100; both Tianjin Damao Chemical Reagent Factory) to yield eleven fractions (CA1-CA11). 11-O-acetylcyathatriol (4.6 g; Fig. 1) was obtained from fraction CA9 by recrystallization in methanol. The purity was determined by normalization of the peak areas in high-performance liquid chromatography (HPLC; LC-20A, Shimadzu Corporation, Kyoto, Japan), and was found to be $99.3 \%$, using an ultraviolet detector (LT28-UV600; Agilent Technologies, Inc., Santa Clara, CA, USA).

Reagents. RPMI-1640 medium and fetal bovine serum (FBS) were obtained from Invitrogen; Thermo Fisher Scientific, Inc. (Waltham, MA, USA). The nitric oxide determination kit (Griess method), mouse tumor necrosis factor (TNF)- $\alpha$ ELISA kit (cat. no. SEM024) and mouse interleukin (IL)-6 ELISA kit (cat. no. SEM008), cell lysis buffer and bicinchoninic acid (BCA) protein concentration assay kit were products of Yantai Science and Biotechnology Co., Ltd. (Yantai, China). Mouse anti-rabbit inducible nitric oxide synthase (iNOS) polyclonal antibody (cat. no. 160862; Cayman Chemical Co., Ann Arbor, MI, USA), mouse anti-rabbit cyclooxygenase-2 (COX-2) polyclonal antibody (cat. no. 160106, Cayman Chemical Co.), goat antirabbit phosphorylated (p) extracellular signal-regulated kinase

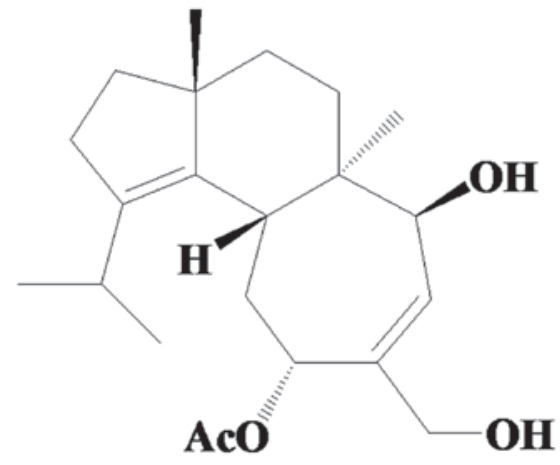

Figure 1. Chemical structure of 11- $O$-acetylcyathatriol.

(ERK 1/2) polyclonal antibody (cat. no. AF1015; Affinity Bio, Scoresby, VIC, Australia), goat anti-rabbit p-c-Jun N-terminal kinase (p-JNK) polyclonal antibody (cat. no. AF3318; Affinity Bio), goat anti-rabbit p-p38 polyclonal antibody (cat. no. AF3455; Affinity Bio), goat anti-rabbit I $\kappa \mathrm{B}-\alpha$ polyclonal antibody (cat. no. sc-371; Santa Cruz Biotechnology, Inc., Dallas, TX, USA), goat anti-rabbit $\beta$-actin polyclonal antibody (cat. no. sc-1616; Santa Cruz Biotechnology, Inc.), horseradish peroxidase (HRP)-conjugated goat anti-rabbit IgG $(\mathrm{H}+\mathrm{L})$ (cat. no. S0001; Affinity Bio) were used at a dilution of 1:1,000. MTT, LPS, hydrocortisone (cat. no. H4001; purity $\geq 98 \%$ by HPLC) and other reagents were purchased from Sigma-Aldrich (St. Louis, MO, USA). Omeprazole enteric-coated capsules (20 mg/capsule; lot. 613071024) was a product of Shandong Luoxin Pharmaceutical Co., Ltd. (Linyi, China).

Culture of the RAW 264.7 cells. RAW 264.7 cells, obtained from American Type Culture Collection (no. TIB-71) were cultured in RPMI-1640 medium supplemented with $10 \%$ heat inactivated FBS at $37^{\circ} \mathrm{C}$ in a humidified incubator. The medium was routinely replaced every 2 days. The RAW 264.7 cells were passaged by trypsinization $[0.25 \%(\mathrm{w} / \mathrm{v})$ trypsin; Yantai Science and Biotechnology Co., Ltd.] until they attained confluence.

Cell viability assay. The 11- $O$-acetylcyathatriol was dissolved in $100 \%$ cell culture grade dimethyl sulfoxide (Yantai Science and Biotechnology Co., Ltd.) at a concentration of $50 \mathrm{mM}$. The stock solution was stored at $-20^{\circ} \mathrm{C}$ and diluted immediately prior to use. The RAW 264.7 cells were adjusted to a density of $1 \times 10^{6} / \mathrm{ml}$ and then $200 \mu \mathrm{l}$ was added into each well of a 96-well plate. The cells were treated with $11-O$-acetylcyathatriol at $0.39,0.78,1.56,3.12,6.25,12.5,25,50$ and $100 \mu \mathrm{M}$, and incubated at $37^{\circ} \mathrm{C}$ for $24 \mathrm{~h}$. The mitochondrial-dependent reduction of MTT to formazan was used to measure cell viability (17). All the experimental procedures were the same as those previously reported (18).

Determination of NO. The RAW 264.7 cells were treated with $1 \mu \mathrm{g} / \mathrm{ml}$ LPS, together with different concentrations $(1.5625$, $3.125,6.25$ and $12.5 \mu \mathrm{M}$ ) of $11-O$-acetylcyathatriol at $37^{\circ} \mathrm{C}$ for $24 \mathrm{~h}$. Subsequently, $100 \mu \mathrm{l}$ of the culture supernatant was removed, and the concentrations of nitrite were determined using an NO determination kit, according to the manufacturer's protocol (19). 
Determination of levels of TNF- $\alpha$ and IL- 6 cytokines. The RAW 264.7 cells were treated with $1 \mu \mathrm{g} / \mathrm{ml}$ LPS, together with different concentrations $(1.5625,3.125,6.25$ and $12.5 \mu \mathrm{M})$ of 11- $O$-acetylcyathatriol at $37^{\circ} \mathrm{C}$ for $6 \mathrm{~h}$. Following treatment, $100 \mu \mathrm{l}$ of the culture supernatant was removed and was used to determine the levels of TNF- $\alpha$ and IL- 6 using respective ELISA assay kits, according to the manufacturer's protocols (20).

Protein extraction. Following the indicated treatments, the medium was removed and the cells were washed with phosphate-buffered saline (PBS). The cells were lysed in $40 \mu \mathrm{l}$ cell lysis buffer by incubation at $4^{\circ} \mathrm{C}$ for $15 \mathrm{~min}$, and then centrifuged at $13,400 \mathrm{xg}$ and $4^{\circ} \mathrm{C}$ for $10 \mathrm{~min}$ to obtain the total protein samples for analysis of iNOS, COX-2, p-ERK1/2, p-JNK, p-p38 and IкB- $\alpha$ using western blot analysis. Total protein concentrations were determined using the BCA protein concentration assay kit, according to the manufacturer's instructions $(18,21)$.

Western blot analysis. Total proteins $(30 \mu \mathrm{g})$ were subjected to appropriate SDS-PAGE and then transferred onto nitrocellulose membranes (Pall Corporation, Port Washington, NY, USA). The membranes were blocked with $5 \%$ skim milk in Tris-buffered saline with $0.05 \%$ (v/v) Tween-20 (TBS-T; Yantai Science and Biotechnology Co., Ltd.) at room temperature for $1 \mathrm{~h}$. Following washing with TBS-T, the membranes were incubated with the respective primary antibody solutions at $4^{\circ} \mathrm{C}$ overnight. The membranes were washed with TBS-T and then incubated in HRP-conjugated secondary antibody solution for $1 \mathrm{~h}$ at room temperature. The membranes were washed with TBS-T and detected using enhanced chemiluminescence (Beyotime Institute of Biotechnology, Shanghai, China). Images were exposed to X-ray films (Kodak, Rochester, NY, USA) and collected, and the respective bands were quantitated by densitometric analysis using DigDoc100 software. The densitometric data of iNOS, COX-2, p-ERK1/2, p-JNK, p-p38 and IкB- $\alpha$ were normalized on the basis of the levels of $\beta$-actin $(18,21)$.

Animal grouping. Male Sprague-Dawley rats weighing 180-220 g were purchased from the Experimental Animal Center of Shandong Luye Pharmaceutical Co., Ltd. [Yantai, China; animal care and use committee approval no. SCXK (Lu) 20090009]. All experimental procedures performed in the present study were performed in accordance with the guidelines for the care and use of laboratory animals, and were approved by the Ethical Committee of Yantai University (Yantai, China; approval no. 201408002; 14th Aug, 2014). A total of 36 rats were randomly divided into six groups: Normal control group, ethanol model group, omeprazole group (positive control group), low dose 11- $O$-acetylcyathatriol group $(5 \mathrm{mg} / \mathrm{kg})$, middle dose 11- $O$-acetylcyathatriol group $(10 \mathrm{mg} / \mathrm{kg})$, high dose $11-O$-acetylcyathatriol group $(15 \mathrm{mg} / \mathrm{kg})$.

Ethanol-induced gastric injury. The rats in the normal control group and ethanol model group were administered with $5 \mathrm{mg} / \mathrm{ml}$ sodium carboxymethyl cellulose $(1 \mathrm{ml} / 100 \mathrm{~g}$ body weight; intragastrically). The rats in the positive control group were administered with omeprazole $(5 \mathrm{mg} / \mathrm{kg} / \mathrm{day})$.
The 11- $O$-acetylcyathatriol treatment groups were administered with respective doses of 11- $O$-acetylcyathatriol (low dose, $5 \mathrm{mg} / \mathrm{kg} / \mathrm{day}$; middle dose, $10 \mathrm{mg} / \mathrm{kg} / \mathrm{day}$; high dose, $15 \mathrm{mg} / \mathrm{kg} /$ day). The drugs were suspended in $5 \mathrm{mg} / \mathrm{ml}$ sodium carboxymethyl cellulose and administered intragastrically for 10 days $(1 \mathrm{ml} / 100 \mathrm{~g}$ body weight, once each day). At $2 \mathrm{~h}$ following the final administration, the ethanol model group and the treatment groups were administered with $75 \%$ ethanol ( $1 \mathrm{ml} / 200 \mathrm{~g}$ intragastrically) to induce gastric injury, and specimens were collected after $1 \mathrm{~h}$.

Specimen collection and handling. Following intraperitoneal anesthesia with $10 \%$ chloral hydrate $(0.35 \mathrm{ml} / 100 \mathrm{~g})$, an incision was made along the greater curvature of the stomach following ligation of the cardia and pylorus of the stomach. The stomach was rinsed with normal saline, and pathological changes in the gastric mucosa were examined and recorded using the gastric ulcer index (UI), which was calculated using the Guth method (22). The stomach was fixed with $10 \%$ formaldehyde (Yantai Science and Biotechnology Co., Ltd.), and the same area of the gastric mucosa was then embedded in paraffin (Yantai Science and Biotechnology Co., Ltd.), sectioned (size, $5 \mu \mathrm{m})$ using a microtome and stained with hematoxylin and eosin (Yantai Science and Biotechnology Co., Ltd.). The pathological features were determined by analysis under a microscope (MM-40; Nikon Corporation, Tokyo, Japan). The animals were sacrificed by air embolism.

UI score calculation. Damage $\leq 1 \mathrm{~mm}$, including erosion point, was assigned a score of 1 . Injury $\leq 2 \mathrm{~mm}$ was assigned a score of 2 . Injury $\leq 3 \mathrm{~mm}$ was assigned a score of 3 . Injury $\leq 4 \mathrm{~mm}$ was assigned a score of 4 , and damage $>4 \mathrm{~mm}$ was assigned a score of 5 . The scores were added together to obtain the UI. The ulcer inhibition rate was calculated as follows: Ulcer inhibition rate $(\%)=100 \% \mathrm{x}$ (UI of model group - UI of drug treatment group) / UI of model group.

Statistical analysis. Data were analyzed using SPSS 13.0 software (SPSS, Inc., Chicago, IL, USA). The results are expressed as the mean \pm standard deviation. Statistical comparisons were conducted using a post hoc test subsequent to one-way analysis of variance and $\mathrm{P}<0.05$ was considered to indicate a statistically significant difference.

\section{Results and Discussion}

The RAW 264.7 cells were treated with different concentrations of 11- $O$-acetylcyathatriol for $24 \mathrm{~h}$, and the cell viability was then assessed using the MTT method. As shown in Fig. 2A, 11- $O$-acetylcyathatriol did not show any significant cytotoxic effects at concentrations between 0.39 and $25 \mu \mathrm{M}$ against the RAW 264.7 cells.

The RAW 264.7 cells were treated with $1 \mu \mathrm{g} / \mathrm{ml}$ LPS with or without indicated concentrations of 11-O-acetylcyathatriol $(1.5625,3.125,6.25$ and $12.5 \mu \mathrm{M})$ or the positive control, hydrocortisone $(100 \mu \mathrm{M})$, for $24 \mathrm{~h}$. The concentrations of $\mathrm{NO}_{2}$ in the supernatant were then measured as an indicator of $\mathrm{NO}$ production. As shown in Fig. 2B, 11- $O$-acetylcyathatriol significantly suppressed the LPS-induced production of NO, and its inhibitory activity was equally potent, compared with 
A
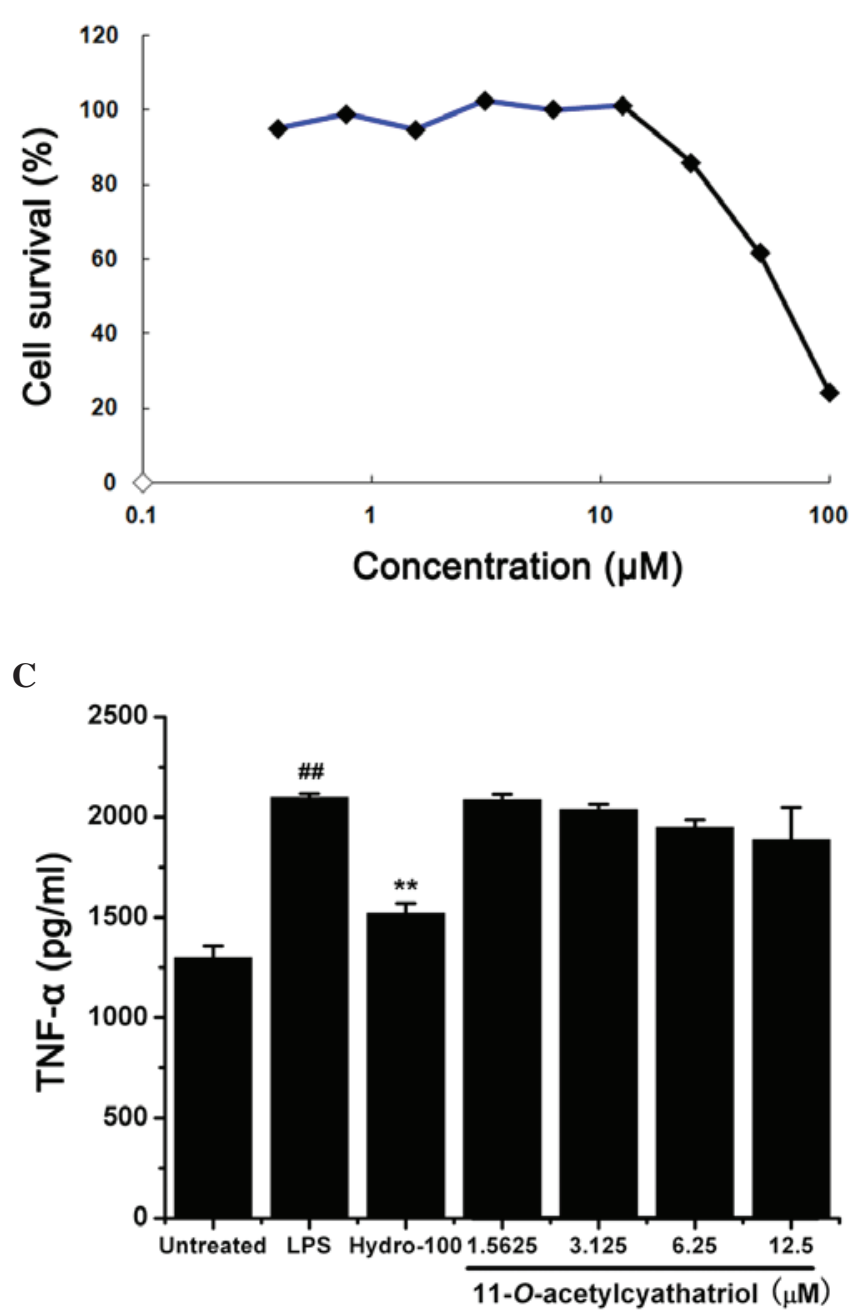

B

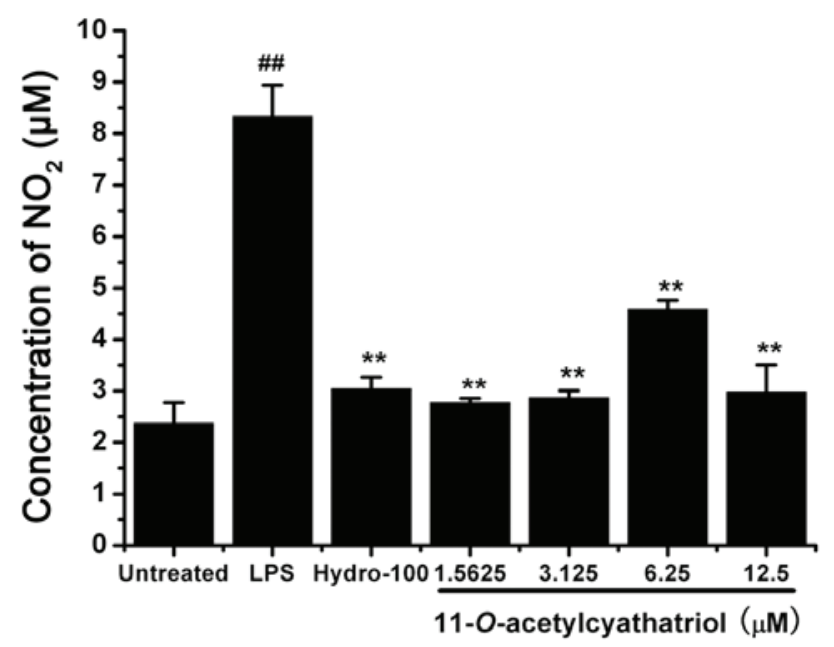

D

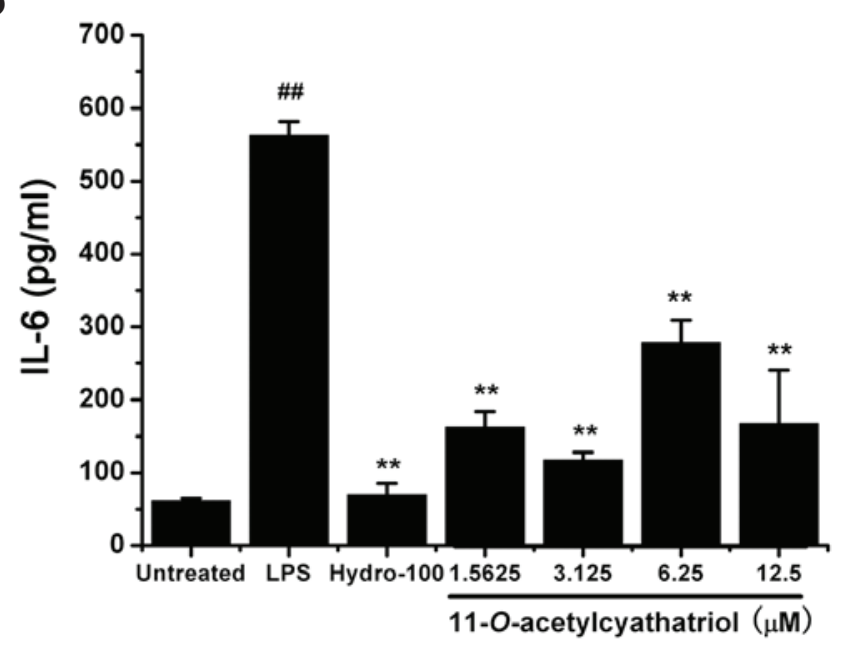

Figure 2. Cytotoxic effects of 11- $O$-acetylcyathatriol against RAW 264.7 cells and its effects on TNF- $\alpha$, IL-6 and NO. (A) Cytotoxicity of 11- $O$-acetylcyathatriol against RAW 264.7 cells. Cells were treated with 11-O-acetylcyathatriol at concentrations of $0.39,0.78,1.56,3.125,6.25,12.5,25,50$ and $100 \mu \mathrm{M}$ for $24 \mathrm{~h}$. The cell viability was evaluated using an MTT assay. (B) Effects of 11- $O$-acetylcyathatriol on NO secretion. RAW 264.7 cells were treated with $1 \mu \mathrm{g} / \mathrm{ml} \mathrm{LPS}$ with either indicated concentrations of $11-O$-acetylcyathatriol $(1.5625,3.125,6.25$ and $12.5 \mu \mathrm{M})$ or hydrocortisone $(100 \mu \mathrm{M})$ for $24 \mathrm{~h}$. Culture supernatant $(100 \mu 1)$ was removed to determine the concentration of $\mathrm{NO}_{2}{ }^{-}$. The experiment was performed in triplicate, and the results are expressed as the mean \pm standard deviation from three separate experiments $(\mathrm{n}=3)$. ${ }^{* *} \mathrm{P}<0.01$, vs. LPS group; ${ }^{* \#} \mathrm{P}<0.01$, vs. untreated group. Effects of $11-O$-acetylcyathatriol on the the release of (C) TNF- $\alpha$ and (D) IL-6. The RAW 264.7 cells were treated with $1 \mu \mathrm{g} / \mathrm{ml}$ of LPS with either the indicated concentrations of 11-O-acetylcyathatriol (1.5625, $3.125,6.25$ and $12.5 \mu \mathrm{M})$ or hydrocortisone $(100 \mu \mathrm{M})$ for $6 \mathrm{~h}$. Culture supernatant (100 $\mu \mathrm{l})$ was removed to determine the levels of TNF- $\alpha$ and IL-6 using respective ELISA assay kits, according to the manufacturer's protocols. The experiment was performed in triplicate and the results are expressed as the mean \pm standard deviation from three separate experiments $(n=3) .{ }^{* *} \mathrm{P}<0.01$, vs. LPS group; ${ }^{\# \#} \mathrm{P}<0.01$, vs. untreated group. LPS, lipopolysaccharide; NO, nitric oxide; TNF- $\alpha$, tumor necrosis factor- $\alpha$; IL-6, interleukin-6; Hydro, hydrocortisone.

the positive control of hydrocortisone, a commonly used anti-inflammatory drug. The RAW 264.7 cells were also treated with $1 \mu \mathrm{g} / \mathrm{ml}$ LPS with or without indicated concentrations of $11-O$-acetylcyathatriol $(1.5625,3.125,6.25$ and $12.5 \mu \mathrm{M})$ or the positive control, hydrocortisone $(100 \mu \mathrm{M})$, for $6 \mathrm{~h}$. The levels of the pro-inflammatory TNF- $\alpha$ and IL- 6 cytokines in the supernatant were detected using respective ELISA kits. As shown in Fig. 2C and D, 11-O-acetylcyathatriol did not exhibit significant inhibition of LPS-induced TNF- $\alpha$ release, but markedly inhibited IL- 6 release. These results showed that 11- $O$-acetylcyathatriol significantly inhibited certain inflammatory mediators, including NO and IL-6, induced by LPS in RAW 264.7 macrophage cells, and this ability may, to a certain extent, underlie its anti-inflammatory activities.

As the excessive production of NO is always associated with high protein expression levels of iNOS, the protein levels of iNOS and COX-2 were detected using western blot analysis. As shown in Fig. 3A, the LPS-induced high protein expression levels of $\mathrm{iNOS}$ and COX-2 were completely inhibited by the high dose of 11-O-acetylcyathatriol $(100 \mu \mathrm{M})$. Mitogen-activated protein kinase (MAPK) cascades have been shown to be important in the transduction of extracellular signals to cellular responses. In mammalian cells, three MAPK families have been clearly characterized: Classical MAPK, also known as ERK, JNK/MAPK (JNK/SAPK) and p38 kinase. The MAPK pathways relay, amplify and integrate signals from a diverse range of stimuli, and elicit appropriate physiological responses, including cellular proliferation, differentiation, inflammatory responses and apoptosis, in mammalian cells. The effect of 11-O-acetylcyathatriol on the phosphorylation of the ERK1/2, JNK, p38 proteins were detected using western blot analysis. As shown in Fig. 3B, 11-O-acetylcyathatriol 
Table I. Gastric mucosal UI and ulcer inhibition rate.

\begin{tabular}{lccc}
\hline Group & Number of cases & UI & Ulcer inhibition rate $(\%)$ \\
\hline Normal control & 6 & 0 & 0 \\
Ethanol model & 6 & $63.00 \pm 11.08$ & - \\
Omeprazole & 6 & $39.00 \pm 8.25^{\mathrm{a}}$ & 38.10 \\
$5 \mathrm{mg} / \mathrm{kg}$ & 6 & $29.17 \pm 12.61^{\mathrm{a}}$ & 53.70 \\
$10 \mathrm{mg} / \mathrm{kg}$ & 6 & $16.83 \pm 6.97^{\mathrm{a}}$ & 73.28 \\
$15 \mathrm{mg} / \mathrm{kg}$ & 6 & $14.33 \pm 5.72^{\mathrm{a}}$ & 77.25 \\
\hline
\end{tabular}

$\mathrm{UI}$ is expressed as the mean \pm standard deviation. UI, Ulcer Index.

A

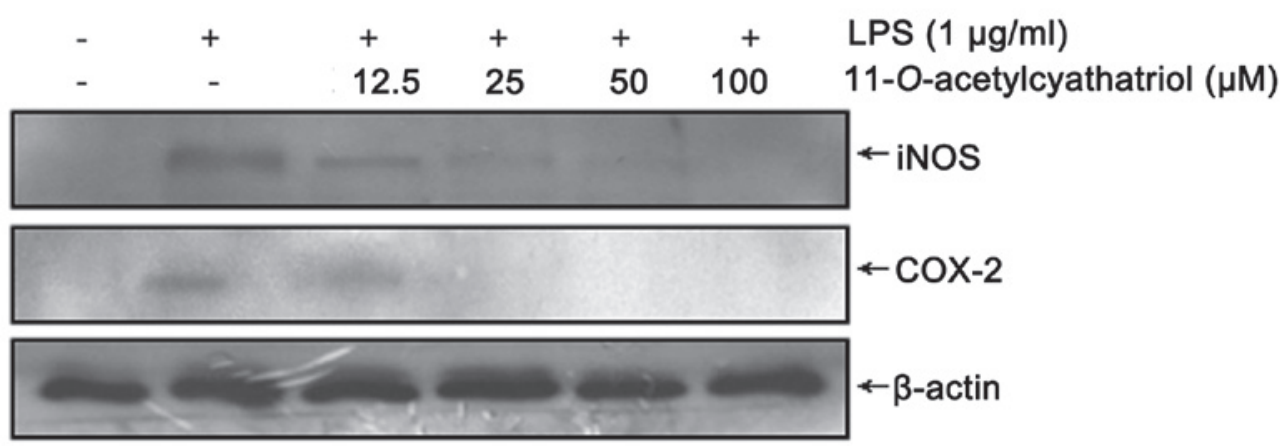

$\mathbf{B}$

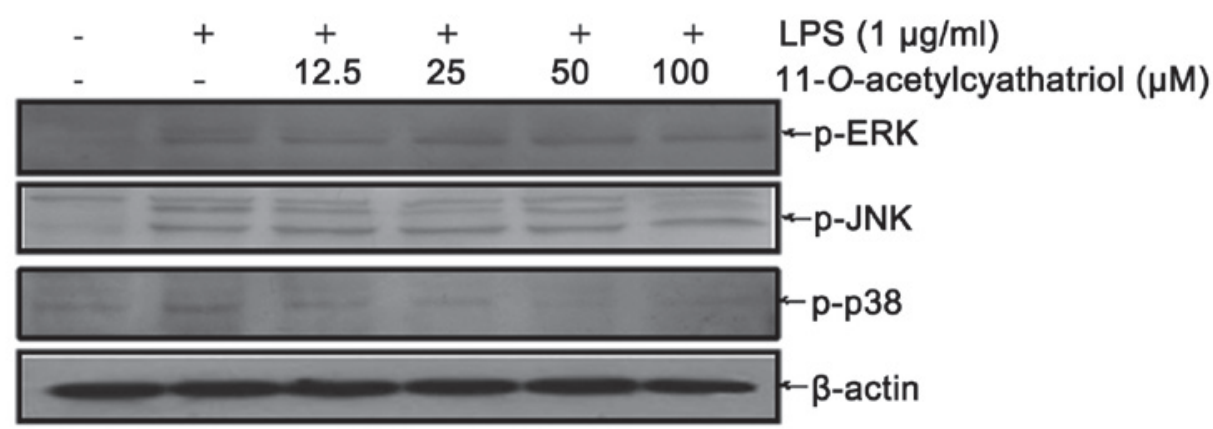

C

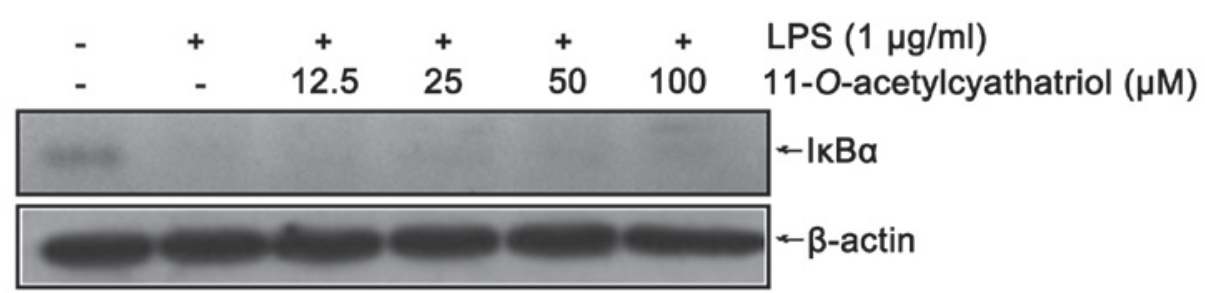

Figure 3. Effects of 11-O-acetylcyathatriol on the protein expression levels of iNOS and COX-2. The RAW 264.7 cells were treated by $1 \mu \mathrm{g} / \mathrm{ml}$ of LPS with indicated concentrations of 11-O-acetylcyathatriol $(12.5,25,50$ and $100 \mu \mathrm{M})$ for $24 \mathrm{~h}$, and the expression levels of (A) iNOS and COX-2 were detected using western blot analysis. (B) Effects of 11- $O$-acetylcyathatriol on the phosphorylation of ERK1/2, JNK and p38 proteins. RAW 264.7 cells were treated with $1 \mu \mathrm{g} / \mathrm{ml}$ LPS with 11- $O$-acetylcyathatriol $(12.5,25,50$ and $100 \mu \mathrm{M})$ for $30 \mathrm{~min}$, and the protein expression levels of p-ERK1/2, p-JNK and p-p38 were detected using western blot analysis. (C) Effects of 11- $O$-acetylcyathatriol on the protein degradation of IкB- $\alpha$. RAW 264.7 cells were treated with $1 \mu \mathrm{g} / \mathrm{ml}$ of LPS with 11- $O$-acetylcyathatriol $(12.5,25,50$ and $100 \mu \mathrm{M})$ for $10 \mathrm{~min}$, and the protein expression of IкB- $\alpha$ was detected using western blot analysis. iNOS, inducible nitric oxide synthase; COX-2, cyclooxygenase-2; LPS, lipopolysaccharide; ERK, extracellular signal-regulated kinase; JNK, c-Jun N-terminal kinase; IкB- $\alpha$, inhibitor of nuclear factor- $\mathrm{kB}-\alpha$; $\mathrm{p}-$, phosphorylated.

treatment markedly inhibited the phosphorylation of the p38 protein induced by LPS, but showed only weak suppression of the phosphorylation of the ERK $1 / 2$ and JNK proteins. The activation of NF- $\mathrm{NB}$ always results from the phosphorylation and degradation of the I $\mathrm{B}-\alpha$ protein. Therefore, the effect of 11-O-acetylcyathatriol on LPS-induced $\mathrm{I} \kappa \mathrm{B}-\alpha$ protein 

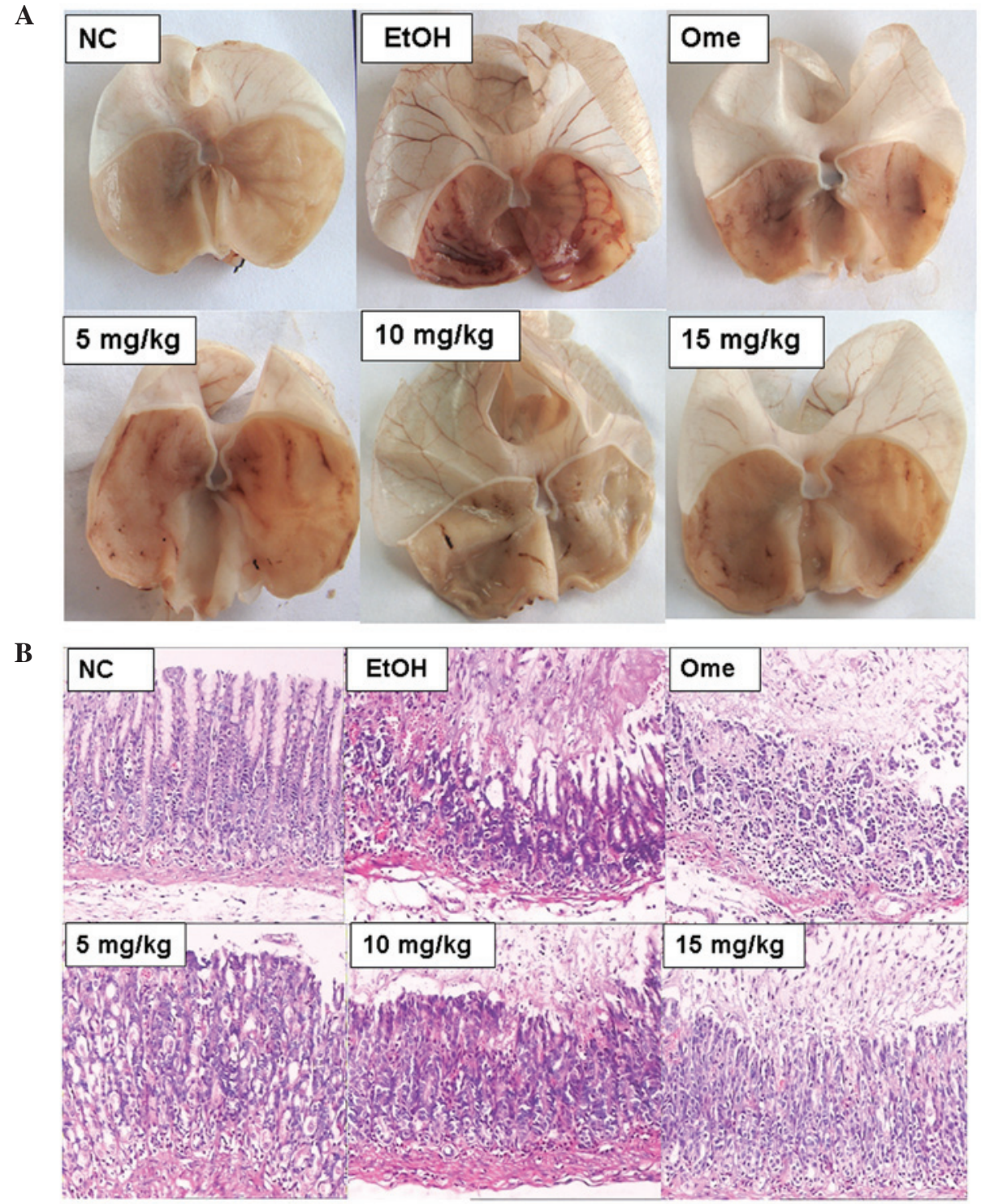

Figure 4. Examination of gastric mucosa injury. (A) Representative images of gastric mucosa. (B) Images to determine histopathological changes in the gastric mucosa (magnification, x100). The severity of gastric mucosal hyperemia, edema, cellular degeneration and necrosis is apparent. NC, normal control group; $\mathrm{EtOH}$, ethanol model group; Ome, $5 \mathrm{mg} / \mathrm{kg}$ omeprazole treatment group; $5 \mathrm{mg} / \mathrm{kg}$, low dose 11- $O$-acetylcyathatriol treatment group; $10 \mathrm{mg} / \mathrm{kg}$, middle dose $11-O$-acetylcyathatriol treatment group; $15 \mathrm{mg} / \mathrm{kg}$, high dose $11-O$-acetylcyathatriol treatment group.

degradation was investigated using western blot analysis. As shown in Fig. 3C, 11-O-acetylcyathatriol had only a weak inhibitory effect on the I $\kappa$ B- $\alpha$ protein degradation induced by LPS.

As is well known, high doses of alcohol are rapidly absorbed in the stomach, directly damaging the gastric mucosa epithelial cells and destroying the gastric mucosa barrier. The local production of increased numbers of inflammatory mediators can cause leukocyte infiltration and an increase in gastric acid secretion, inducing gastric injury, gastritis and gastric ulcer formation. The effect of $11-O$-acetylcyathatriol on alcohol-induced gastric mucosa injury in rats were further investigated in the present study. As shown in Table I, gastric mucosa injury in the rats of the model group (UI score, $63.00 \pm 11.08$ ) was significantly higher, compared with that in the normal control group (UI score, 0 ; $\mathrm{P}<0.01$ ). Omeprazole treatment significantly decreased injury in model group (UI score, $39.00 \pm 8.25$; $\mathrm{P}<0.01$ ). 11- $O$-acetylcyathatriol treat- ment $(5,10$ and $15 \mathrm{mg} / \mathrm{kg})$ exerted significant protective effects on the alcohol-induced gastric mucosa injury, in a dose-dependent manner, compared with the model group $(\mathrm{P}<0.01)$. The ulcer inhibitory rates were $53.70,73.28$ and $77.25 \%$, respectively. As shown in the images in Fig. 4A and B, the severity of gastric mucosal hyperemia, edema, cellular degeneration and necrosis in the 11- $O$-acetylcyathatriol-treatment groups were markedly lower, compared with those in the model group.

In the present study, the possible anti-inflammatory molecular mechanisms of 11-O-acetylcyathatriol, a natural cyathane diterpenoid identified from the medicinal fungus Cyathus africanus, were examined. The 11-O-acetylcyathatriol compound was observed to significantly inhibit various LPS-induced responses of the macrophages, including the overproduction of NO and the release of the pro-inflammatory, cytokine IL-6. Further investigations indicated that $11-O$-acetylcyathatriol downregulated the high protein expression levels of iNOS and COX-2, which also inhibited the activation of the 
MAPK/p-38 signaling transduction pathway. This is the first direct evidence, to the best of our knowledge, to demonstrate the anti-inflammatory effect and molecular mechanism of 11-O-acetylcyathatriol. Taken together, these results may provide the theoretical basis for the use of cyathane diterpenoids, or the use of medicinal plants containing similar constituents and their analogues, in the treatment of inflammatory diseases.

\section{Acknowledgements}

This study was supported by the State Key Laboratory of Mycology, Institute of Microbiology, Chinese Academy of Sciences (Shanghai, China), the Taishan Scholar Project to Fenghua $\mathrm{Fu}$, and the Undergraduate Scientific and Technological Innovation Project of Yantai University (Yantai, China; grant no. 131404).

\section{References}

1. Poinar G Jr: Bird's nest fungi (Nidulariales: Nidulariaceae) in baltic and dominican amber. Fungal Biol 118: 325-329, 2014.

2. WA and Ayer H Taube: Metabolites of Cyathus helenae, Cyathin A3 and allocyathin B3, members of a new group of diterpenoids. Tetrahedron Lett 13: 1917-1920, 1972.

3. Shi XW, Liu L, Gao JM and Zhang AL: Cyathane diterpenes from Chinese mushroom Sarcodon scabrosus and their neurite outgrowth-promoting activity. Eur J Med Chem 46: 3112-3117, 2011

4. Ma BJ and Liu JK: A new bitter diterpenoid from Sarcodon scabrosus. J Basic Microbiol 45: 328-330, 2005.

5. Shen JW, Ruan Y and Ma BJ: Diterpenoids of macromycetes. J Basic Microbiol 49: 242-255, 2009.

6. Wrighf DL and Whitehead CR: Recent progress on the synthesis of cyathane type diterpenes. Org Prep Proced Int 32: 307-330, 2000.

7. Krzyczkowshi W: The structure, medicinal properties and biosynthesis of cyathane diterpenoids. Biotechnologia 1: 146-147, 2008.

8. Ayer WA, Browne LM, Mercer JR, et al: Metabolites of bird's nest fungi. Part 8. Some minor metabolites of Cyathus helenae and some correlations among the cyathins. Can J Chem 56: 717-721, 1978.

9. Ayer WA and Carstens LL: Diterpenoid metabolites of Cyathus helenae, Cyathin B3 and cyathin C3. Can J Chem 51: 3157-3160, 1973.
10. Ayer WA and Taube H: Metabolites of Cyathus helenae. A new class of diterpenoids. Can J Chem 51: 3842-3854, 1973.

11. Ayer WA, Yoshida T and van Schie DMJ: Metabolites of bird's nest fungi. Part 9. Diterpenoid metabolites of Cyathus africanus Brodie. Can J Chem 56: 2113-2120, 1978.

12. Hecht HJ and Striatin ABC: Novel diterpenoid antibiotics from Cyathus striatus; $\mathrm{x}$-ray crystal structure of striatin A. J Chem Soc Chem Commun 15: 665-666, 1978.

13. Ayer WA: Metabolites of bird's nest fungi. Part 11. Diterpenoid metabolites of Cyathus earlei. Can J Chem 57: 3332-3337, 1979.

14. Bai R, Zhang CC, Yin X, Wei J and Gao JM: Striatoids A-F, cyathane diterpenoids with neurotrophic activity from cultures of the fungus Cyathus striatus. J Nat Prod 78: 783-788, 2015.

15. Kang HS, Kim KR, Jun EM, Park SH, Lee TS, Suh JW and Kim JP: Cyathuscavins A, B and C, new free radical scavengers with DNA protection activity from the Basidiomycete Cyathus stercoreus. Bioorg Med Chem Lett 18: 4047-4050, 2008.

16. Han J, Chen Y, Bao L, Yang X, Liu D, Li S, Zhao F and Liu H: Anti-inflammatory and cytotoxic cyathane diterpenoids from the medicinal fungus Cyathus africanus. Fitoterapia 84: 22-31, 2013.

17. Denizot F and Lang R: Rapid colorimetric assay for cell growth and survival. Modifications to the tetrazolium dye procedure giving improved sensitivity and reliability. J Immunol Methods 89: 271-277, 1986.

18. Zhao F, Chen L, Zhang M,Bi C,Li L, Zhang Q, Shi C, Li M,Zhou S and Kong L: Inhibition of lipopolysaccharide-induced iNOS and COX-2 expression by indole alkaloid, 3-(hydroxymethyl)-6,7-dihydroindolo [2,3-a]quinolizin-(12H)- one, via NF- $\mathrm{B}$ inactivation in RAW 264.7 macrophages. Planta Med 79: 782-787, 2013.

19. Ishihara T, Kohno K, Ushio S, Iwaki K, Ikeda M and Kurimoto M: Tryptanthrin inhibits nitric oxide and prostaglandin E (2) synthesis by murine macrophages. Eur J Pharmacol 407: 197-204, 2000.

20. Zhao F, Xu H, He EQ, Jiang YT and Liu K: Inhibitory effects of sesquiterpenes from Saussurea lappa on the overproduction of nitric oxide and TNF-alpha release in LPS-activated macrophages. J Asian Nat Prod Res 10: 1045-1053, 2008.

21. Zhao F, Gao Z, Jiao W, Chen L, Chen L and Yao X: In vitro anti-inflammatory effects of beta-carboline alkaloids, isolated from Picrasma quassioides, through inhibition of the iNOS pathway. Planta Med 78: 1906-1911, 2012.

22. Jeon WY, Lee MY, Shin IS, Lim HS and Shin HK: Protective effects of the traditional herbal formula oryeongsan water extract on ethanol-induced acute gastric mucosal injury in rats. Evid Based Complement Alternat Med 2012: 438191, 2012. 